\title{
SIZE DISTRIBUTIONS OF SOLAR FLARES AND SOLAR ENERGETIC PARTICLE EVENTS
}

\author{
E. W. Cliver ${ }^{1}$, A. G. Ling $^{2}$, A. Belov ${ }^{3}$, and S. Yashiro 4,5 \\ ${ }^{1}$ Space Vehicles Directorate, Air Force Research Laboratory, Sunspot, NM 88349, USA \\ ${ }^{2}$ Atmospheric Environmental Research, Lexington, MA 02421, USA \\ ${ }^{3}$ IZMIRAN, Troitsk, Moscow Region 142190, Russia \\ ${ }^{4}$ NASA Goddard Space Flight Center, Greenbelt, MD 20771, USA \\ 5 Department of Physics, The Catholic University of America, Washington, DC 20064, USA \\ Received 2012 July 10; accepted 2012 August 7; published 2012 August 21
}

\begin{abstract}
We suggest that the flatter size distribution of solar energetic proton (SEP) events relative to that of flare soft X-ray (SXR) events is primarily due to the fact that SEP flares are an energetic subset of all flares. Flares associated with gradual SEP events are characteristically accompanied by fast $\left(\geqslant 1000 \mathrm{~km} \mathrm{~s}^{-1}\right)$ coronal mass ejections (CMEs) that drive coronal/interplanetary shock waves. For the 1996-2005 interval, the slopes ( $\alpha$ values) of power-law size distributions of the peak 1-8 A fluxes of SXR flares associated with (a) $>10 \mathrm{MeV}$ SEP events (with peak fluxes $\geqslant 1 \mathrm{pr} \mathrm{cm}^{-2} \mathrm{~s}^{-1} \mathrm{sr}^{-1}$ ) and (b) fast CMEs were $\sim 1.3-1.4$ compared to $\sim 1.2$ for the peak proton fluxes of $>10 \mathrm{MeV}$ SEP events and $\sim 2$ for the peak 1-8 $\AA$ fluxes of all SXR flares. The difference of $\sim 0.15$ between the slopes of the distributions of SEP events and SEP SXR flares is consistent with the observed variation of SEP event peak flux with SXR peak flux.
\end{abstract}

Key words: Sun: flares - Sun: particle emission - Sun: coronal mass ejections (CMEs) - Sun: X-rays, gamma-rays

\section{INTRODUCTION}

Hudson (1978) pointed out that the size distribution of solar energetic proton (SEP) events is flatter than those of flare electromagnetic emissions. When size distributions are represented by power laws, of the form

$$
d N / d x \sim x^{-\alpha} \text { events/unit } x,
$$

SEP events are found to have values of $\alpha$ that range from $\sim 1.2$ to 1.4 versus values of $\sim 1.8$ to 2.0 for flare electromagnetic emissions. Table 1 contains representative reported values of $\alpha$ for the two types of emissions. The SEP distributions are relatively independent of the energy range considered and the flare source longitude (Belov et al. 2007).

The cause of the difference between the slopes of the distributions in Table 1 is not well understood. Schrijver et al. (2012) recently listed four possible contributors to the discrepancy, including "(1) SEP spectral distributions may depend on event energy (which could include a dependence on the partitioning between flare radiative and coronal mass ejection, CME, bulk-kinetic energies), (2) background corrections, (3) effects of compound events involving two or more $\mathrm{CME} /$ shocks ..., and (4) particle propagation effects in the heliosphere." Schrijver et al. (2012) considered a fifth, geometrical, possibility, the dilution of SEP fluence over an opening angle into the heliosphere and calculated that this effect might be primarily responsible for the observed drop off or "kink" in the $>10 \mathrm{MeV}$ SEP fluence size distribution at a level of $\sim 5-10 \times 10^{9} \mathrm{pr} \mathrm{cm}^{-2}$ first reported by Lingenfelter \& Hudson (1980).

As an alternative explanation for the difference in the powerlaw slopes (values of $\alpha$ ) of the distributions in Table 1, Hudson (1978) suggested that peak proton flux $\left(J_{\mathrm{P}}\right)$ varied with the total flare energy ( $W$; assumed to scale with peak soft X-ray, SXR, flux) as $J_{\mathrm{P}} \sim W^{\beta}$ with $\beta>1$. A determination of $\beta$ from the known values of $\alpha$ in Table 1 for SXR events $\left(\alpha_{\mathrm{X}}=1.84\right.$; Drake $1971)$ and SEP events $\left(\alpha_{\mathrm{P}}=1.15 \pm 0.05\right.$; Van Hollebeke et al.
1975) using the formula

$$
\beta=\left(\alpha_{\mathrm{X}}-1\right) /\left(\alpha_{\mathrm{P}}-1\right)
$$

yielded $\beta=5.6(+2.8,-1.4)$. Hudson suggested that "proton flares probably do not represent a separate category of solar flares, but stand out principally because proton emission strongly biases the observer toward the more energetic events."

In the same year as Hudson's paper, Kahler et al. (1978) presented the first direct evidence that SEP events were associated with CMEs, indicating that proton flares did, in fact, represent a separate class of flares which in time came to be known as eruptive flares to distinguish them from compact or confined events that lacked CMEs (Švestka \& Cliver 1992). Current thinking is that large "gradual" SEP events are caused by fast CMEs that drive coronal/interplanetary shocks manifested by radio type II bursts (Reames 1999; Gopalswamy et al. 2002; Cliver et al. 2004). In this paper, we use this modern view of SEP flares to revisit the cause of the difference in the slopes of the size distributions of solar flare electromagnetic emissions and SEP events.

Our analysis is presented in Section 2 and results are summarized and discussed in Section 3.

\section{ANALYSIS}

From lists of SEP events for solar cycle 23 compiled by Belov et al. (2005, 2007) and Cane et al. (2010), we made a list of all $>10 \mathrm{MeV}$ proton events from 1996 to 2005 that were associated with flares with nominally good (W20-W85) magnetic connection to Earth. To focus on gradual SEP events, we limited the sample to events with hourly averaged peak fluxes $\geqslant 1 \mathrm{pr} \mathrm{cm}^{-2} \mathrm{~s}^{-1} \mathrm{sr}^{-1}$ (or $\geqslant 1$ proton flux unit (pfu)). Cliver (2009) suggested that the upper limit peak flux for the smaller "impulsive" SEP events is $\sim 3$ pfu at this energy (see Cliver \& Cane 2002 for a discussion of SEP event terminology). We only considered the prompt component of each SEP event, ignoring delayed peaks associated with interplanetary shocks. 


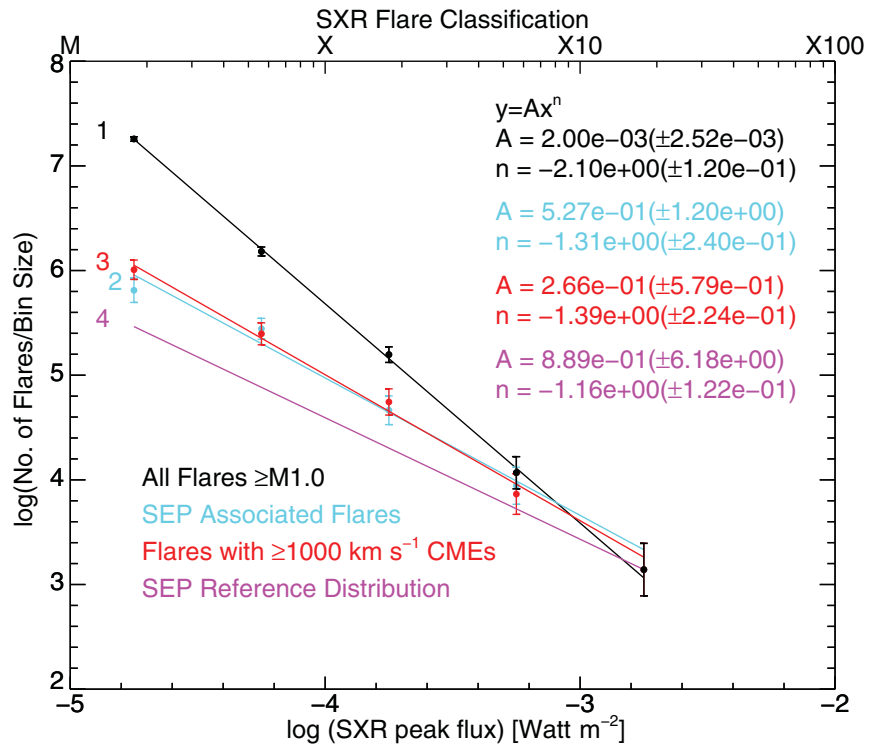

Figure 1. Size distributions for (1) peak 1-8 fluxes of all $\geqslant$ M1.0 SXR flares (black line); (2) peak 1-8 fluxes of SXR flares associated with $>10 \mathrm{MeV}$ proton events with peak fluxes $\geqslant 1$ pfu (light blue line); (3) peak $1-8 \AA$ fluxes of SXR flares associated with $\geqslant 1000 \mathrm{~km} \mathrm{~s}^{-1}$ CMEs (red line); and (4) peak proton fluxes of $>10 \mathrm{MeV}$ SEP events (reference distribution for slope only, magenta line). The SXR flare classification is given at the top of the figure. All distributions are based on flares or SEP events that originated in a longitude range from W20 to W85 (1996-2005).

Table 1

Values of $\alpha$ for SEP and Flare Size Distributions

\begin{tabular}{lcc}
\hline \hline Parameter & Value of $\alpha$ & Reference \\
\hline SEPs & & \\
20-80 MeV Flux & $1.15 \pm 0.05$ & Van Hollebeke et al. (1975) \\
$>10 \mathrm{MeV}$ Fluence & $1.24 \pm 0.04$ & Gabriel \& Feynman (1996) \\
$>10 \mathrm{MeV}$ Flux & $1.37 \pm 0.03$ & Belov et al. (2005) \\
Flare radiation & & \\
Hard X-rays & $1.73 \pm 0.01$ & Crosby et al. (1993) \\
Microwaves & $1.73 \pm 0.04$ & Nita et al. (2004) \\
Soft X-rays & $1.98 \pm 0.11$ & Aschwanden \& Freeland (2012) \\
\hline
\end{tabular}

We subtracted the pre-event background for both the 1-8 $\AA$ SXR bursts and $>10 \mathrm{MeV}$ SEP events (extrapolating the time profile of any preceding SEP event to the time of the peak of the event). The list of 58 SEP events with their associated SXR and CME data is given in Table 2 .

Figure 1 contains power-law size distributions for (1) the peak 1-8 A fluxes of 540 SXR flares of class $\geqslant$ M1.0 $(\geqslant 1.0 \times$ $10^{-5} \mathrm{~W} \mathrm{~m}^{-2}$ ) (black line; data from Yashiro et al. 2006, http://cdaw.gsfc.nasa.gov/pub/yashiro/flare_cme/fclist_pub.txt); (2) the peak $1-8 \AA$ fluxes of $52 \geqslant$ M1.0 SXR flares associated with $>10 \mathrm{MeV}$ proton events (light blue line; Table 2); (3) the peak 1-8 fluxes of 59 SXR flares associated with CMEs with speeds $\geqslant 1000 \mathrm{~km} \mathrm{~s}^{-1}$ (red line; Yashiro et al. 2006); and (4) the peak proton fluxes of $58>10 \mathrm{MeV}$ SEP events (reference distribution for slope only, magenta line; Table 2). The $1000 \mathrm{~km} \mathrm{~s}^{-1}$ CME speed threshold corresponds roughly to the speeds of CMEs required to produce type II bursts that will extend from the metric to the kilometric wavelength range (Gopalswamy et al. 2005). Such shocks from western-hemisphere flares are highly associated with large SEP events (Gopalswamy et al. 2008).

To facilitate comparisons, and minimize SEP propagation and SXR occultation effects, all of the size distributions in

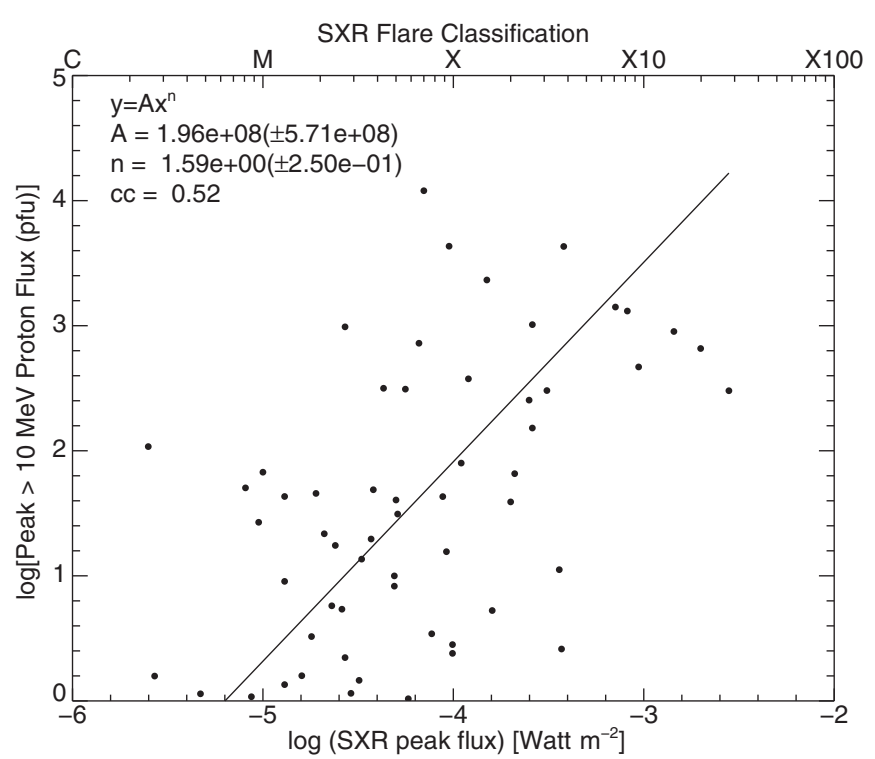

Figure 2. Scatter plot of peak $>10 \mathrm{MeV} \mathrm{SEP} \mathrm{event} \mathrm{flux} \mathrm{vs.} \mathrm{flare} \mathrm{peak} 1-8 \AA$ flux for proton events originating from W20 to W85 heliolongitude (1996-2005), with geometric mean regression line.

Figure 1 are based on flares or SEP events that originated in a longitude range from W20 to W85 for the period from 1996 to 2005. Distributions 2-4 are based on background (preevent) corrected SXR and SEP data. No background subtraction was made for distribution 1 but the $\alpha$ value of $2.10 \pm 0.12$ is comparable to that $(1.98 \pm 0.11)$ recently obtained in the comprehensive study of Aschwanden \& Freeland (2012; Table 1). The smaller slope $(1.73 \pm 0.01)$ of the hard X-ray flare distribution (Crosby et al. 1993) is attributed to the fact that big flares produce relatively more hard X-rays (e.g., Battaglia et al. 2005). The slopes of the distributions based on uncorrected data (for size distributions $2(1.28 \pm 0.24), 3(1.38 \pm 0.22)$, and $4(1.15 \pm 0.12))$ differ little from those of the backgroundcorrected distributions shown in Figure 1.

Figure 1 shows that the slopes of size distributions 2 and 3 , for the peak 1-8 A fluxes of SEP- and fast-CME-associated flares, respectively, are significantly flatter than that for the peak $1-8 \AA$ Aluxes of all flares. The similarity of distributions 2 and 3 reflects substantial overlap in the two databases; $61 \%(36 / 59)$ of the fast-CME-flares were associated with SEP events in Table 1 and $75 \%(41 / 55)$ of the SEP flares (no CME coverage for the three events) in Table 1 had associated fast CMEs. The median CME speed of the 14 events with speeds $<1000 \mathrm{~km} \mathrm{~s}^{-1}$ was $806 \mathrm{~km} \mathrm{~s}^{-1}$ (range from 464 to $998 \mathrm{~km} \mathrm{~s}^{-1}$ ). The $\alpha$ values of these two distributions (SEP flares: $\alpha=1.31 \pm 0.24$; fast CMEs: $1.39 \pm 0.22)$ are close to that of the $>10 \mathrm{MeV}$ SEPs during this period (distribution 4: $1.16 \pm 0.12$ ).

Inserting the value of $\alpha_{\mathrm{X}}=1.31 \pm 0.24$ from distribution 2 for the SXR peak fluxes of SEP flares into Equation (2) and using $\alpha_{\mathrm{P}}=1.16 \pm 0.12$ gives a value for $\beta$ of $1.94(+11.9,-1.69)$. The 1.94 base value of $\beta$ is similar to that obtained for the regression line in the scatter plot in Figure 2 between the peak proton fluxes of the $58>10 \mathrm{MeV}$ SEP events in Table 2 and their associated peak 1-8 $\mathrm{A}$ SXR fluxes $(1.59 \pm 0.25$; background subtracted for both parameters). The regression line in Figure 2 was determined by assuming that the uncertainties in the logs of the $>10 \mathrm{MeV}$ flux and the SXR intensity are comparable, an assumption we believe is justified because the approximately three-order-of-magnitude scatter in CME energy for a given 
Table 2

SEP Events with Peak Fluxes $\geqslant 1$ pfu (1996-2005)

\begin{tabular}{|c|c|c|c|c|c|c|c|c|}
\hline \multirow[b]{2}{*}{$\begin{array}{l}\text { Date } \\
\text { (yyyy/mm/dd) }\end{array}$} & \multicolumn{5}{|c|}{ SXR } & \multicolumn{2}{|c|}{$>10 \mathrm{MeV}$} & \multirow{2}{*}{$\begin{array}{c}\text { CME } \\
\text { Speed } \\
\left(\mathrm{km} \mathrm{s}^{-1}\right)\end{array}$} \\
\hline & $\begin{array}{l}\text { Onset } \\
\text { Time } \\
\text { (UT) }\end{array}$ & $\begin{array}{l}\text { Peak } \\
\text { Time } \\
\text { (UT) }\end{array}$ & $\begin{array}{l}\text { Initial } \\
\text { Class }\end{array}$ & $\begin{array}{c}\text { BKG Corr. } \\
\text { Class }\end{array}$ & $\begin{array}{c}\text { Solar } \\
\text { Lat/Lon }\end{array}$ & $\begin{array}{l}\text { Peak } \\
\text { Flux } \\
(\mathrm{pfu})\end{array}$ & $\begin{array}{c}\text { BKG Corr. } \\
\text { Flux } \\
\text { (pfu) }\end{array}$ & \\
\hline $1997 / 11 / 04$ & $05: 52$ & $05: 58$ & X 2.1 & X 2.1 & S14W33 & $6.59 \mathrm{e}+01$ & $6.56 \mathrm{e}+01$ & 785 \\
\hline $1997 / 11 / 06$ & $11: 49$ & $11: 55$ & X 9.4 & X 9.4 & S18W63 & $4.69 e+02$ & $4.68 \mathrm{e}+02$ & 1556 \\
\hline $1998 / 05 / 06$ & $07: 58$ & 08:09 & X 2.7 & X 2.6 & S11W65 & $1.52 \mathrm{e}+02$ & $1.52 \mathrm{e}+02$ & 1099 \\
\hline $1998 / 09 / 30$ & $13: 08$ & $13: 50$ & M 2.8 & M 2.7 & N19W85 & $9.79 \mathrm{e}+02$ & $9.79 \mathrm{e}+02$ & d.g. \\
\hline $1998 / 11 / 07$ & $11: 02$ & $11: 06$ & M 2.4 & M 2.3 & N14W43 & $6.46 e+00$ & $5.76 \mathrm{e}+00$ & 632 \\
\hline $1998 / 11 / 22$ & $06: 30$ & $06: 42$ & X 3.7 & X 3.7 & S27W82 & $2.85 \mathrm{e}+00$ & $2.60 \mathrm{e}+00$ & d.g. \\
\hline $1999 / 06 / 04$ & $06: 52$ & 07:03 & M 3.9 & M 3.8 & N17W69 & $5.08 \mathrm{e}+01$ & $4.88 \mathrm{e}+01$ & 2230 \\
\hline $2000 / 02 / 12$ & $03: 51$ & 04:10 & M 1.7 & M 1.6 & N26W24 & $1.74 \mathrm{e}+00$ & $1.59 \mathrm{e}+00$ & 1107 \\
\hline $2000 / 04 / 04$ & $15: 12$ & $15: 41$ & C 9.7 & C 8.1 & N16W66 & $5.07 e+01$ & $5.05 \mathrm{e}+01$ & 1188 \\
\hline $2000 / 05 / 15$ & $15: 46$ & $16: 01$ & C 7.8 & C 4.7 & S22W68 & $1.30 \mathrm{e}+00$ & $1.14 \mathrm{e}+00$ & 1212 \\
\hline $2000 / 06 / 10$ & $16: 40$ & $17: 02$ & M 5.2 & M 5.0 & N22W40 & $4.07 e+01$ & $4.04 \mathrm{e}+01$ & 1108 \\
\hline $2000 / 06 / 18$ & $01: 52$ & 01:59 & X 1.0 & M 9.9 & N23W85 & $3.07 e+00$ & $2.82 \mathrm{e}+00$ & 629 \\
\hline $2000 / 06 / 23$ & $14: 18$ & $14: 31$ & M 3.0 & M 2.9 & N23W72 & $1.31 \mathrm{e}+00$ & $1.15 \mathrm{e}+00$ & 847 \\
\hline $2000 / 06 / 25$ & $07: 17$ & $07: 52$ & M 1.9 & M 1.8 & N16W55 & $3.42 \mathrm{e}+00$ & $3.27 \mathrm{e}+00$ & 1617 \\
\hline $2000 / 07 / 22$ & $11: 17$ & $11: 34$ & M 3.7 & M 3.3 & N14W56 & $1.54 \mathrm{e}+01$ & $1.36 \mathrm{e}+01$ & 1230 \\
\hline $2000 / 11 / 08$ & $22: 42$ & $23: 28$ & M 7.4 & M 7.0 & N10W75 & $1.20 \mathrm{e}+04$ & $1.20 \mathrm{e}+04$ & 1732 \\
\hline $2001 / 01 / 28$ & $15: 40$ & $16: 00$ & M 1.5 & M 1.3 & S04W59 & $4.33 e+01$ & $4.31 \mathrm{e}+01$ & 916 \\
\hline 2001/04/02 & $10: 58$ & $11: 36$ & X 1.1 & M 9.9 & N16W62 & $3.17 \mathrm{e}+00$ & $2.40 \mathrm{e}+00$ & 992 \\
\hline 2001/04/02 & $21: 32$ & $21: 51$ & $\mathrm{X} 20.0$ & X19.9 & N17W78 & $6.59 \mathrm{e}+02$ & $6.57 \mathrm{e}+02$ & 2505 \\
\hline $2001 / 04 / 12$ & 09:39 & $10: 28$ & X 2.0 & X 2.0 & S20W42 & $4.40 \mathrm{e}+01$ & $3.90 \mathrm{e}+01$ & 1184 \\
\hline $2001 / 04 / 15$ & $13: 19$ & $13: 50$ & X14.4 & X14.4 & S20W84 & $9.00 \mathrm{e}+02$ & $8.99 \mathrm{e}+02$ & 1199 \\
\hline $2001 / 04 / 26$ & $11: 26$ & $13: 12$ & M 7.8 & M 7.7 & N17W27 & $3.63 e+00$ & $3.44 \mathrm{e}+00$ & 1006 \\
\hline $2001 / 06 / 04$ & $16: 11$ & $16: 33$ & C 3.2 & C 2.7 & N23W60 & $1.91 \mathrm{e}+00$ & $1.58 \mathrm{e}+00$ & 464 \\
\hline $2001 / 09 / 15$ & 11:04 & $11: 28$ & M 1.5 & M 1.3 & S24W51 & $9.20 \mathrm{e}+00$ & $9.03 e+00$ & 478 \\
\hline $2001 / 10 / 19$ & $16: 13$ & $16: 30$ & X 1.6 & X 1.6 & N15W30 & $9.68 \mathrm{e}+00$ & $5.28 \mathrm{e}+00$ & 901 \\
\hline $2001 / 11 / 22$ & $20: 18$ & $20: 36$ & M 3.8 & M 3.7 & S24W68 & $2.00 \mathrm{e}+01$ & $1.97 \mathrm{e}+01$ & 1443 \\
\hline $2001 / 11 / 22$ & $22: 32$ & $23: 30$ & M 9.9 & M 9.5 & S15W34 & $4.34 \mathrm{e}+03$ & $4.32 \mathrm{e}+03$ & 1437 \\
\hline $2001 / 12 / 26$ & $04: 32$ & $05: 40$ & M 7.1 & M 6.6 & N08W54 & $7.24 \mathrm{e}+02$ & $7.24 \mathrm{e}+02$ & 1446 \\
\hline $2002 / 02 / 20$ & $05: 52$ & $06: 12$ & M 5.1 & M 4.9 & N12W72 & $8.44 \mathrm{e}+00$ & $8.27 \mathrm{e}+00$ & 952 \\
\hline $2002 / 04 / 17$ & $07: 46$ & $08: 24$ & M 2.6 & M 2.4 & S14W36 & $1.76 \mathrm{e}+01$ & $1.75 \mathrm{e}+01$ & 1240 \\
\hline $2002 / 04 / 21$ & $00: 43$ & 01:51 & X 1.5 & X 1.5 & S14W84 & $2.32 \mathrm{e}+03$ & $2.32 \mathrm{e}+03$ & 2393 \\
\hline $2002 / 05 / 22$ & $03: 18$ & $03: 54$ & C 5.0 & C 2.5 & S22W53 & $1.08 \mathrm{e}+02$ & $1.08 \mathrm{e}+02$ & 1557 \\
\hline $2002 / 08 / 14$ & $01: 47$ & $02: 12$ & M 2.3 & M 2.1 & N09W54 & $2.19 \mathrm{e}+01$ & $2.17 \mathrm{e}+01$ & 1309 \\
\hline $2002 / 08 / 20$ & $08: 22$ & $08: 26$ & M 3.4 & M 3.2 & S11W38 & $1.77 e+00$ & $1.46 \mathrm{e}+00$ & 1099 \\
\hline $2002 / 08 / 22$ & 01:47 & $01: 57$ & M 5.4 & M 5.1 & S07W62 & $3.13 \mathrm{e}+01$ & $3.12 \mathrm{e}+01$ & 998 \\
\hline $2002 / 08 / 24$ & 00:49 & 01:12 & X 3.1 & X 3.1 & S02W81 & $3.03 e+02$ & $3.03 e+02$ & 1913 \\
\hline 2002/11/09 & 13:08 & $13: 23$ & M 4.6 & M 4.3 & S12W29 & $3.16 \mathrm{e}+02$ & $3.16 \mathrm{e}+02$ & 1838 \\
\hline $2003 / 05 / 28$ & $00: 17$ & $00: 27$ & X 3.6 & X 3.6 & S06W20 & $1.13 \mathrm{e}+01$ & $1.12 \mathrm{e}+01$ & 1366 \\
\hline $2003 / 05 / 31$ & $02: 13$ & $02: 24$ & M 9.3 & M 9.2 & S07W65 & $1.58 \mathrm{e}+01$ & $1.56 \mathrm{e}+01$ & 1835 \\
\hline $2003 / 10 / 26$ & $17: 21$ & $18: 19$ & X 1.2 & X 1.2 & N02W38 & $3.76 e+02$ & $3.76 \mathrm{e}+02$ & 1537 \\
\hline $2003 / 11 / 02$ & $17: 03$ & $17: 25$ & X 8.3 & X 8.2 & S14W56 & $1.31 \mathrm{e}+03$ & $1.31 \mathrm{e}+03$ & 2598 \\
\hline $2003 / 11 / 03$ & 01:09 & 01:30 & X 2.7 & X 2.6 & N10W83 & $1.42 \mathrm{e}+03$ & $1.02 \mathrm{e}+03$ & 827 \\
\hline $2003 / 11 / 04$ & $19: 29$ & $19: 44$ & X28.0 & X28.0 & S19W83 & $3.03 e+02$ & $3.02 \mathrm{e}+02$ & 2657 \\
\hline 2004/04/11 & $03: 54$ & 04:19 & C 9.6 & C 9.5 & S14W47 & $2.70 \mathrm{e}+01$ & $2.68 \mathrm{e}+01$ & 1645 \\
\hline $2004 / 07 / 25$ & $14: 19$ & $15: 14$ & M 1.1 & M 1.0 & N08W33 & $6.76 e+01$ & $6.74 \mathrm{e}+01$ & 1333 \\
\hline 2004/09/19 & $16: 46$ & $17: 12$ & M 1.9 & M 1.9 & N03W58 & $4.58 \mathrm{e}+01$ & $4.56 \mathrm{e}+01$ & d.g. \\
\hline $2004 / 10 / 30$ & $16: 18$ & $16: 33$ & M 5.9 & M 5.8 & N15W20 & $1.94 \mathrm{e}+00$ & $1.04 \mathrm{e}+00$ & 690 \\
\hline 2004/11/09 & $16: 59$ & $17: 19$ & M 8.9 & M 8.8 & N07W51 & $6.80 \mathrm{e}+01$ & $4.30 \mathrm{e}+01$ & 2000 \\
\hline $2004 / 11 / 10$ & 01:59 & $02: 13$ & X 2.5 & X 2.5 & N09W49 & $2.66 e+02$ & $2.54 \mathrm{e}+02$ & 3387 \\
\hline $2005 / 01 / 17$ & $06: 59$ & $09: 52$ & X 3.8 & X 3.8 & N15W25 & $4.41 e+03$ & $4.30 \mathrm{e}+03$ & 2094 \\
\hline $2005 / 01 / 19$ & 08:03 & $08: 22$ & X 1.3 & X 1.1 & N19W47 & $1.10 \mathrm{e}+02$ & $7.97 \mathrm{e}+01$ & 2020 \\
\hline $2005 / 01 / 20$ & $06: 36$ & 07:01 & X 7.1 & X 7.1 & N14W61 & $1.44 \mathrm{e}+03$ & $1.41 \mathrm{e}+03$ & 882 \\
\hline $2005 / 05 / 06$ & 03:05 & $03: 14$ & C 9.3 & C 8.7 & S06W74 & $1.30 \mathrm{e}+00$ & $1.08 \mathrm{e}+00$ & 1120 \\
\hline $2005 / 05 / 06$ & $11: 11$ & $11: 28$ & M 1.3 & M 1.3 & S05W80 & $1.85 e+00$ & $1.35 \mathrm{e}+00$ & 1144 \\
\hline 2005/07/09 & $21: 47$ & $22: 06$ & M 2.8 & M 2.7 & N11W27 & $2.39 \mathrm{e}+00$ & $2.22 \mathrm{e}+00$ & 1540 \\
\hline $2005 / 07 / 13$ & 14:01 & $14: 49$ & M 5.0 & M 4.9 & N10W80 & $1.02 \mathrm{e}+01$ & $9.99 \mathrm{e}+00$ & 1423 \\
\hline $2005 / 08 / 22$ & $00: 44$ & 01:33 & M 2.6 & M 2.6 & S09W48 & $5.63 e+00$ & $5.41 \mathrm{e}+00$ & 1194 \\
\hline $2005 / 08 / 22$ & $16: 46$ & $17: 27$ & M 5.6 & M 5.6 & S12W60 & $3.11 \mathrm{e}+02$ & $3.11 \mathrm{e}+02$ & 2378 \\
\hline
\end{tabular}


peak SXR flux (Yashiro \& Gopalswamy 2008) is similar to the range in the scatter of SEP peak flux with CME speed (Kahler 2001). Applying a solar longitude correction factor (Kahler 1982) to the peak SEP fluxes in Figure 2 had a negligible effect on the slope of the regression line; it yielded a $\beta$ of $1.61 \pm 0.23$.

\section{SUMMARY AND DISCUSSION}

We have presented evidence that the difference in the slopes of the power-law size distributions of solar flares and SEP events arises primarily because SEP flares, in particular those associated with gradual SEP events, represent an energetic subset of all flares, characterized by fast $\left(\geqslant 1000 \mathrm{~km} \mathrm{~s}^{-1}\right)$ CMEs. The slope ( $\alpha$ value) of the size distribution for the peak 1-8 $\AA$ fluxes of SEP- (or fast CME-) associated SXR flares is 1.3-1.4, compared to $\sim 1.2$ for the peak proton fluxes of $>10 \mathrm{MeV}$ SEP events and $\sim 2$ for the peak 1-8 $\AA$ fluxes of all SXR flares (Figure 1). The remaining difference in the slopes of the peak flux distributions of SEP flares and SEP events is consistent with the observed variation of SEP peak flux with SXR peak flux (Figure 2). This second aspect of the analysis is poorly constrained, however, and all or part of the remaining difference could result from a propagation effect such as the dilution of SEP flux with opening angle proposed by Schrijver et al. (2012). In either case, it is clear that much of the discrepancy in the slopes of the size distributions of SEP events and SEP flares can be accounted for by the energetic nature of gradual SEP flares in relation to all flares.

Yashiro et al. (2006) determined power-law slopes for distributions of the peak 1-8 $\AA$ fluxes of different classes of SXR flares as follows: flares without CMEs $(\alpha=2.52 \pm 0.03)$, all flares $(2.16 \pm 0.03)$, and flares with CMEs of any speed $(1.98 \pm$ $0.05)$. The further decrease in $\alpha$ that we obtained for flares with $\geqslant 1000 \mathrm{~km} \mathrm{~s}^{-1}$ CMEs was expected. For type II bursts, Pearson et al. (1989) constructed histograms of the peak counting rates of hard X-ray bursts with and without associated metric type II bursts. These histograms have $\alpha \sim 1.2$ for the type-II-associated events and $\sim 2$ for the non-type-II events. Gopalswamy et al. (2005) showed that a hierarchal relationship exists between the frequency range of type II bursts and the speeds/energies of associated CMEs: (1) metric only type IIs have a mean CME speed of $610 \mathrm{~km} \mathrm{~s}^{-1}$, (2) metric to decametric/hectometric type II bursts are associated with CMEs with a mean speed of $1115 \mathrm{~km} \mathrm{~s}^{-1}$, and (3) metric to kilometric type IIs have a mean CME speed of $1490 \mathrm{~km} \mathrm{~s}^{-1}$. Belov et al. (2007) were the first to draw attention to the similarity of the size distributions of SEP flares and SEP events.

In regard to SEPs, there are three kinds of flares: (1) those that produce large "gradual" events (Reames 1999; Cliver 2009); (2) those that produce small "impulsive" SEP events with abundance anomalies for ${ }^{3} \mathrm{He}$ and high- $Z$ elements (Reames et al. 1985; Mason et al. 1986; Reames \& Ng 2004); and (3) confined flares that do not produce escaping particles. The inferred magnetic topologies of these three kinds of flares are quite different, involving the pinching off of a rising loop for gradual events (e.g., Forbes 2000), interchange reconnection between open and closed field lines for impulsive events (e.g., Shimojo \& Shibata 2000; Kahler et al. 2001), and field relaxation within a closed flux tube or interaction between closed flux tubes for confined events. What is remarkable is that these disparate types of flares combine to form a single well-defined powerlaw size distribution over several orders of magnitude in SXR intensity.

E.W.C. thanks Karel Schrijver and Jürg Beer for organizing a series of stimulating ISSI workshops on Extreme Solar Events. We thank Hugh Hudson and Karel Schrijver for helpful comments on the manuscript. E.W.C. was supported under AFOSR Task 2301RDZ4 and A.G.L. acknowledges support from AFRL contract FA8718-05-C-0036.

\section{REFERENCES}

Aschwanden, M. J., \& Freeland, S. L. 2012, ApJ, 754, 112

Battaglia, M., Grigis, P.C., \& Benz, A.O. 2005, A\&A, 439, 737

Belov, A., Garcia, H., Kurt, V., Mavromichalaki, H., \& Gerontidou, M. 2005, Sol. Phys., 229, 135

Belov, A., Kurt, V., Mavromichalaki, H., \& Gerontidou, M. 2007, Sol. Phys., 246,457

Cane, H. V., Richardson, I. G., \& von Rosenvinge, T. T. 2010, J. Geophys. Res., 115, A08101

Cliver, E. W., Kahler, S. W., \& Reames, D. V. 2004, ApJ, 605, 902

Cliver, E. W., \& Cane, H. V. 2002, Eos Trans. AGU, 83, 61

Cliver, E. W. 2009, Cent. Eur. Astrophys. Bull., 33, 253

Crosby, N. B., Aschwanden, M. J., \& Dennis, B. R. 1993, Sol. Phys., 143, 275

Drake, J. F. 1971, Sol. Phys., 16, 152

Forbes, T. G. 2000, J. Geophys. Res., 105, 23153

Gabriel, S. B., \& Feynman, J. 1996, Sol. Phys., 165, 337

Gopalswamy, N., Aguilar-Rodriguez, E., Yashiro, S., et al. 2005, J. Geophys. Res., 110, A12S07

Gopalswamy, N., Yashiro, S., Akiyama, S., et al. 2008, Ann. Geophys., 26, 3033

Gopalswamy, N., Yashiro, S., Michalek, G., et al. 2002, ApJ, 572, L103

Hudson, H. S. 1978, Sol. Phys., 57, 237

Kahler, S. W. 1982, J. Geophys. Res., 87, 3439

Kahler, S. W. 2001, J. Geophys. Res., 106, 20947

Kahler, S. W., Hildner, E., \& Van Hollebeke, M. A. I. 1978, Sol. Phys., 57, 429

Kahler, S. W., Reames, D. V., \& Sheeley, N. R., Jr. 2001, ApJ, 562, 558

Lingenfelter, R. E., \& Hudson, H. S. 1980, in The Ancient Sun, ed. R. O. Pepin, J. A. Eddy, \& R. B. Merrill (New York: Pergamon), 69

Mason, G. M., Reames, D. V., von Rosenvinge, T. T., Klecker, B., \& Hovestadt, D. 1986, ApJ, 303, 849

Nita, G. M., Gary, D. E., \& Jeongwoo, L. 2004, ApJ, 605, 528

Pearson, D. H., Nelson, R., Kojoian, G., \& Seal, J. 1989, ApJ, 336, 1050

Reames, D. V. 1999, Space Sci. Rev., 90, 413

Reames, D. V., \& Ng, C. K. 2004, ApJ, 610, 510

Reames, D. V., von Rosenvinge, T. T., \& Lin, R. P. 1985, ApJ, 292, 716

Schrijver, C.J., Beer, J., Baltensperger, U., et al. 2012, J. Geophys. Res., in press (2012arXiv1206.4889S)

Shimojo, M., \& Shibata, K. 2000, ApJ, 542, 1100

Švestka, Z., \& Cliver, E. W. 1992, in IAU Colloq. 133, Eruptive Solar Flares, Vol. 399, ed. Z. Švestka, B. V. Jackson, \& M. E. Machado (New York: Springer), 1

Van Hollebeke, M. A. I., Ma Sung, L. S., \& McDonald, F. B. 1975, Sol. Phys., 41,189

Yashiro, S., Akiyama, S., Gopalswamy, N., \& Howard, R. A. 2006, ApJ, 650, L143

Yashiro, S., \& Gopalswamy, N. 2008, in IAU Symp. 257, Universal Heliophysical Processes, ed. N. Gopalswamy \& D. F. Webb (Cambridge: Cambridge Univ. Press), 233 